Supporting Information for

\title{
Development of a microfluidic-based post-treatment process for size-controlled lipid nanoparticles and application to siRNA delivery
}

Niko Kimura, ${ }^{a \#}$ Masatoshi Maeki,,${ }^{b, \#^{*}}$ Yusuke Sato, ${ }^{d}$ Akihiko Ishida,${ }^{b}$ Hirofumi Tani, ${ }^{b}$ Hideyoshi Harashima, ${ }^{d}$ and Manabu Tokeshi ${ }^{\text {be, } f^{*}}$

${ }^{a}$ Graduate School of Chemical Sciences and Engineering, Hokkaido University, Kita 13 Nishi 8, Kita-ku, Sapporo, 060-8628, Japan

${ }^{b}$ Division of Applied Chemistry, Faculty of Engineering, Hokkaido University, Kita 13 Nishi 8, Kita-ku, Sapporo 060-8628, Japan

cJST PRESTO, 4-1-8 Honcho, Kawaguchi, Saitama, 332-0012, Japan

${ }^{d}$ Faculty of Pharmaceutical Sciences, Hokkaido University, Kita 12 Nishi 6, Kita-ku, Sapporo 060-0812, Japan

'Innovative Research Center for Preventive Medical Engineering, Nagoya University, Furo-cho, Chikusa-ku, Nagoya 464-8601, Japan

Institute of Nano-Life Systems, Institutes of Innovation for Future Society, Nagoya University, Furo-cho, Chikusa-ku, Nagoya 464-8601, Japan

\# N.K. and M.M contributed equally to this work. 
*Corresponding authors:

Masatoshi Maeki E-mail address: m.maeki@eng.hokudai.ac.jp Tel: +81-11-706-6745 Fax: +81-11-706-6745

Manabu Tokeshi E-mail address: tokeshi@eng.hokudai.ac.jp Tel: +81-11-706-6744 Fax: +81-11-706-6745 


\section{Number-weighted size distributions of the POPC LNPs produced at different}

post-treatment conditions and the conventional methods
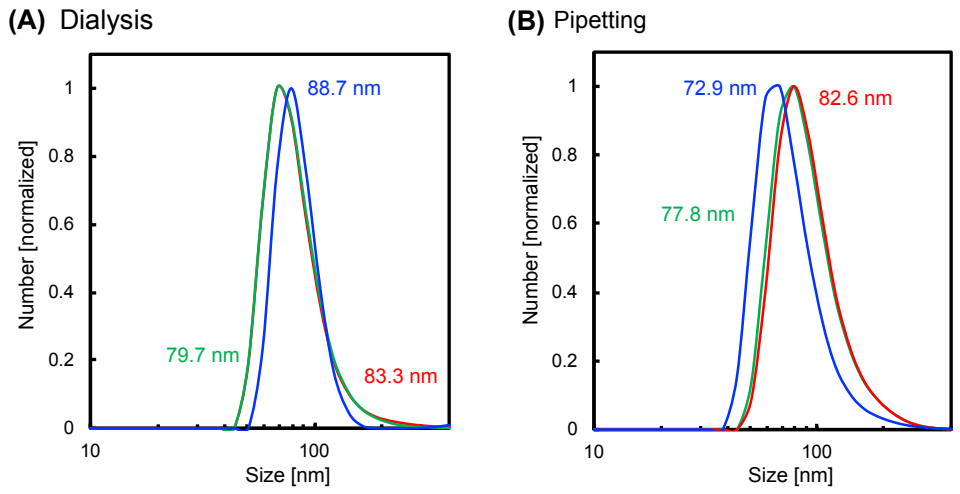

(C) $0.1 \mu \mathrm{L} / \mathrm{min}$

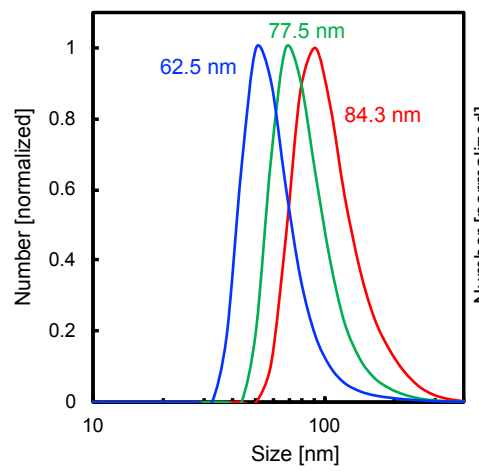

(F) $300 \mu \mathrm{L} / \mathrm{min}$

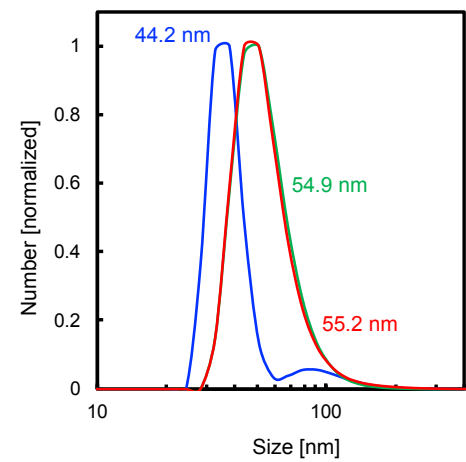

(D) $10 \mu \mathrm{L} / \mathrm{min}$

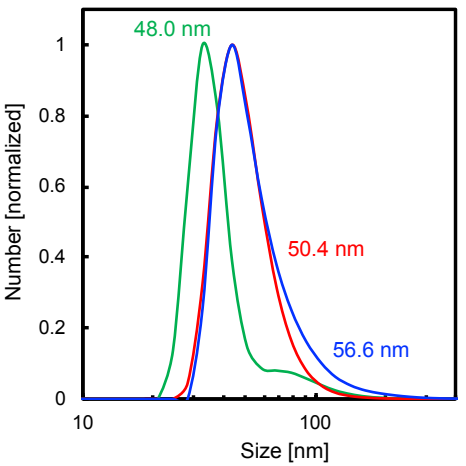

(E) $100 \mu \mathrm{L} / \mathrm{min}$

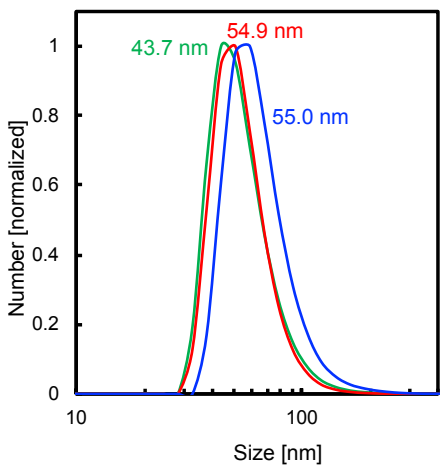

(G) $500 \mu \mathrm{L} / \mathrm{min}$

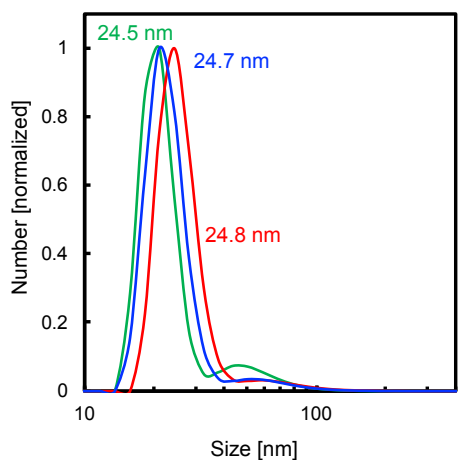

Figure S1. Number weighted size distributions of the POPC LNPs treated by (A, B) the conventional methods and $(\mathrm{C}-\mathrm{G})$ the on-device post-treatment at the different flow rate 
conditions. Number-weighed LNP sizes acquired from the Zetasizer Nano ZS ZEN3600 instrument were shown in each graph. 


\section{Effects of the microfluidic post-treatment process on DPPC LNPs}

1,2-Dipalmitoyl-sn-glycero-3-phosphocholine (DPPC), cholesterol and 1,2-distearoylsn-glycero-3-phosphoethanolamine-N-[amino(polyethylene glycol)-2000] (DSPEPEG2K) were dissolved in ethanol at the molar ratio of $67 / 33 / 5$, and the total lipid concentration was $15 \mathrm{mM}$. The lipid solution and PBS were introduced into the baffle device at the total flow rate of $50 \mu \mathrm{L} / \mathrm{min}$ and the flow rate ratio (FFR) of 3 . The collected suspension containing $25 \% \mathrm{v} / \mathrm{v}$ ethanol was post-treated by the conventional methods (the dialysis method and the direct dilution with a buffer solution method) and the microfluidic method. For the microfluidic method, the LNP suspension containing $25 \% \mathrm{v} / \mathrm{v}$ ethanol was diluted from $25 \%$ to $1 \%$ at $500 \mu \mathrm{L} / \mathrm{min}$ and the FRR of 24 . In the direct dilution method, the suspension was also diluted from $25 \%$ to $1 \%$ ethanol. After the post-treatment, the sizes of LNPs were measured by dynamic light scattering (DLS) measurement.

Figure S2 (A) shows number-weighted size distributions of the LNPs prepared by the conventional and microfluidic post-treatment methods. The size of the LNPs prepared by the microfluidic post-treatment method was the smallest among the three and this trend was seen with high reproducibility (Figure S2 (B)). 
(A)

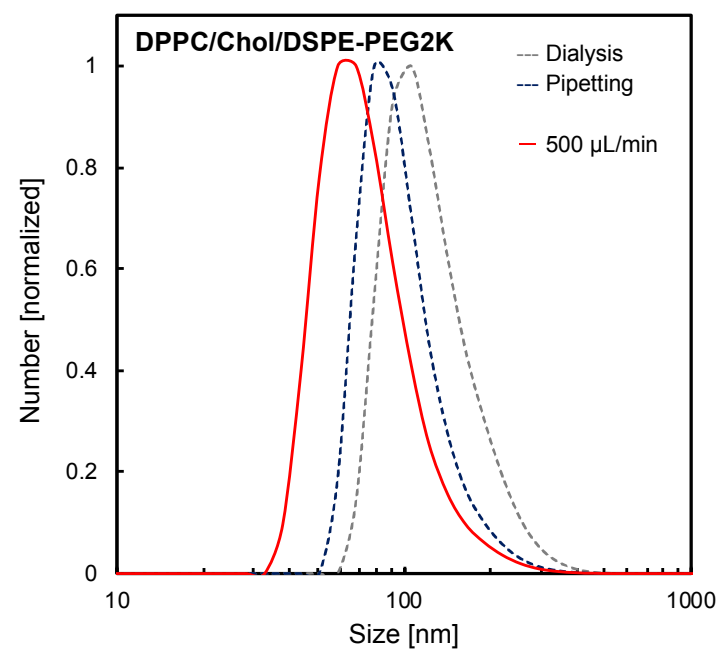

(B)

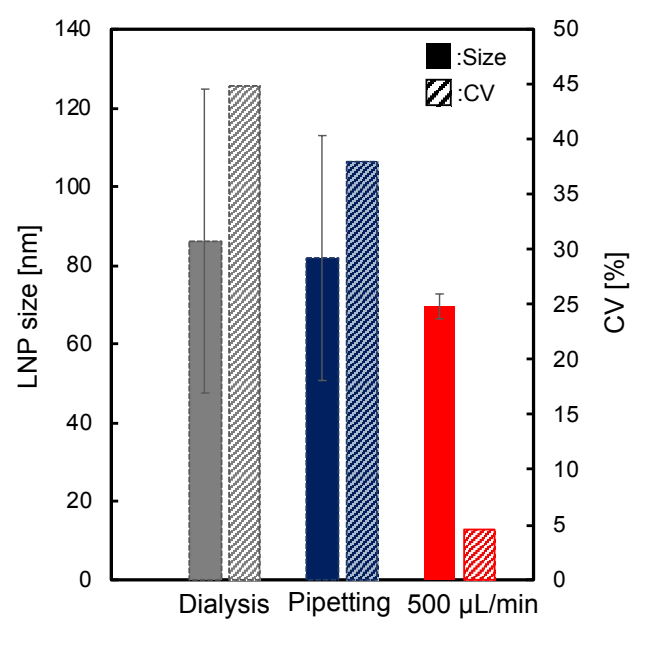

Figure S2. Effects of the post-treatment process on the DPPC-LNP size controllability.

(A) Number-weighted size distributions of the LNPs prepared by the conventional and microfluidic post-treatment methods. (B) Average sizes of LNPs (number-weighted) and CV values. These error bars were calculated from reproducibility experiments carried out three times. 


\section{Evaluation of the size stability of the post-treated POPC LNPs}

We prepared the LNP suspension with $13.4 \mathrm{mM}$ POPC/ethanol solution and saline by using the simple baffle device at the total flow rate of $50 \mu \mathrm{L} / \mathrm{min}$ and the FRR of 3 . The POPC LNP suspension was treated by the conventional post-treatment methods and the on-device method. In the on-device post-treatment method, the POPC LNP suspension was diluted at the total flow rate of $500 \mu \mathrm{L} / \mathrm{min}$. After each post-treatment processes, these treated LNP suspension was incubated at $4{ }^{\circ} \mathrm{C}$ for 10 days. We measured the size of the LNPs before and after the incubation by DLS. Figure S3 shows number-weighted size distributions of the POPC LNPs. The size distributions did not change, regardless of the post-treatment methods. We confirmed the LNPs showed good size stability at least for 10 days.

(A)

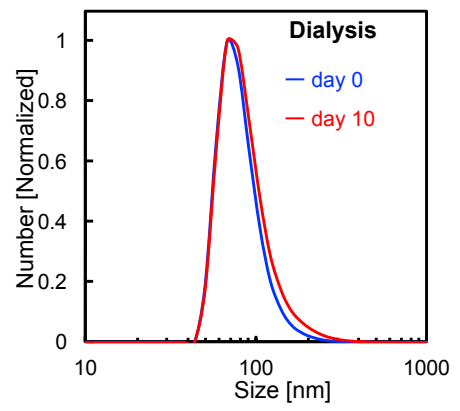

(B)

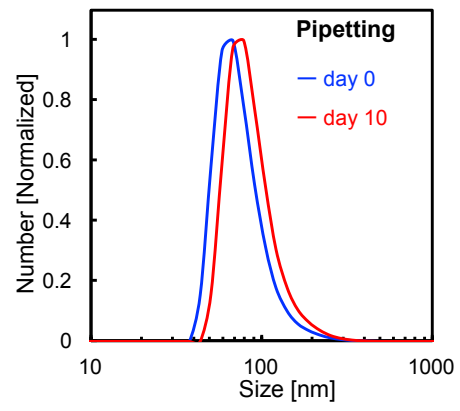

(C)

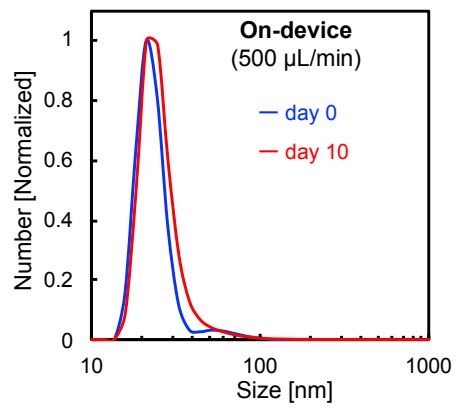

Figure S3. Number-weighted size distributions of the LNPs treated by the conventional and the on-device post-treatment methods. The POPC LNP was synthesized at the total flow rate of $50 \mu \mathrm{L} / \mathrm{min}$ and FRR of 3 . The total flow rate of the on-device method was $500 \mu \mathrm{L} / \mathrm{min}$. After the post-treatment method, the LNPs were incubated at $4^{\circ} \mathrm{C}$ for 10 
days (red). These distributions were normalized by the respective maximum intensity value. 


\section{TEM image analysis of POPC LNPs}

(A)

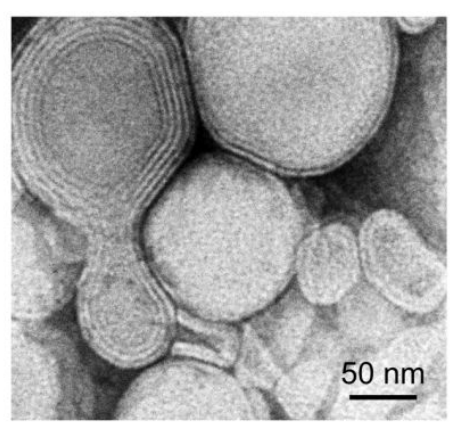

(C)

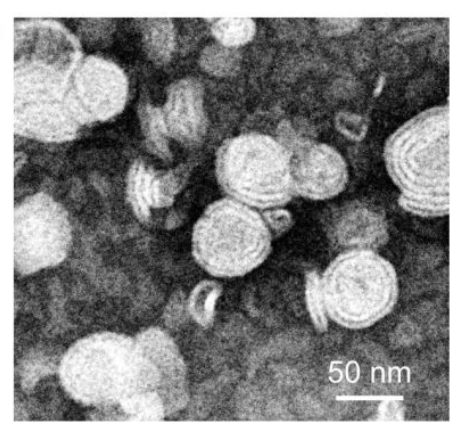

(B)

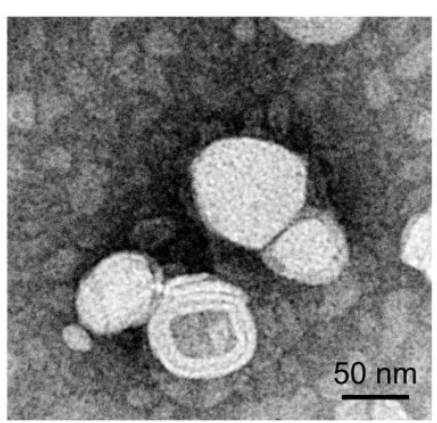

(D)

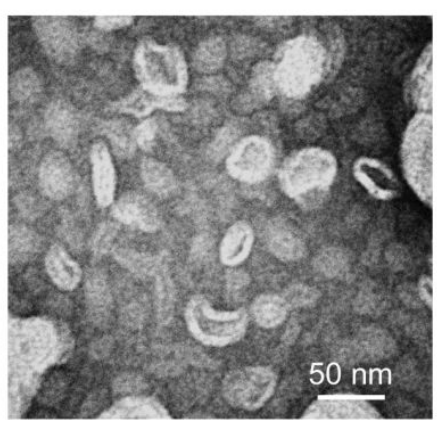

Figure S4. TEM images of the POPC LNPs (A) suspended in 25\% ethanol, (B) treated by the conventional dialysis post-treatment. (C and D) The POPC LNPs treated by the microfluidic post-treatments at the total flow rate of (C) $10 \mu \mathrm{L} / \mathrm{min}$ and (D) $500 \mu \mathrm{L} / \mathrm{min}$. 


\section{Investigation of the shearing force induced size change of the prepared POPC}

\section{LNPs}

We prepared the LNP suspension with $13.4 \mathrm{mM}$ POPC/ethanol solution and saline by using the simple baffle device at the total flow rate of $50 \mu \mathrm{L} / \mathrm{min}$ and the FRR of 3 . The prepared suspension of POPC LNPs $(25 \% \mathrm{v} / \mathrm{v}$ remaining ethanol) was introduced into this baffle device at the flow rate of $500 \mu \mathrm{L} / \mathrm{min}$ without additional ethanol dilution. The reintroduced POPC LNP suspension was collected and dialyzed against saline (Figure S5 (A)). Otherwise, the dialyzed POPC LNP suspension was introduced into the simple baffle device and the collected LNPs were measured by DLS (Figure S5 (B)).

(A)
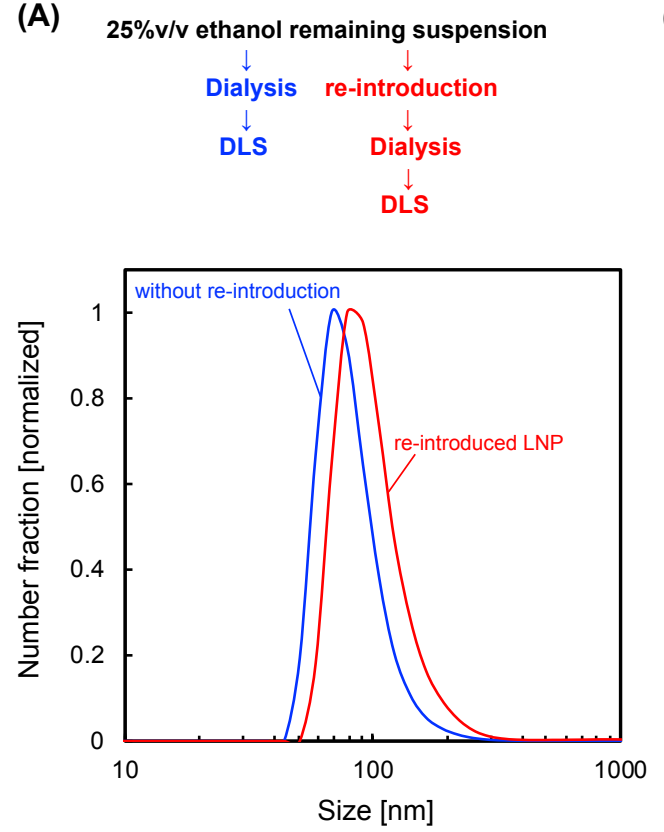

(B)
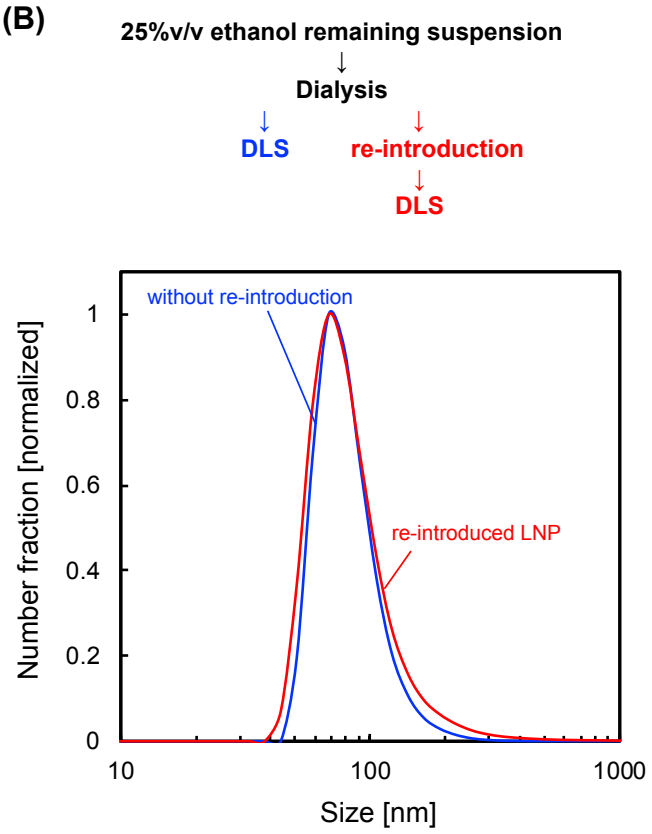
Figure S5. LNP size distributions. (A) The collected LNP suspension with 25\% v/v remaining ethanol was dialyzed after the preparation (blue) or re-introduced into the simple baffle device (red). (B) The dialyzed LNP suspension was introduced again into the simple baffle device. 


\section{Evaluation of the size distribution of the LNPs with the NTA}

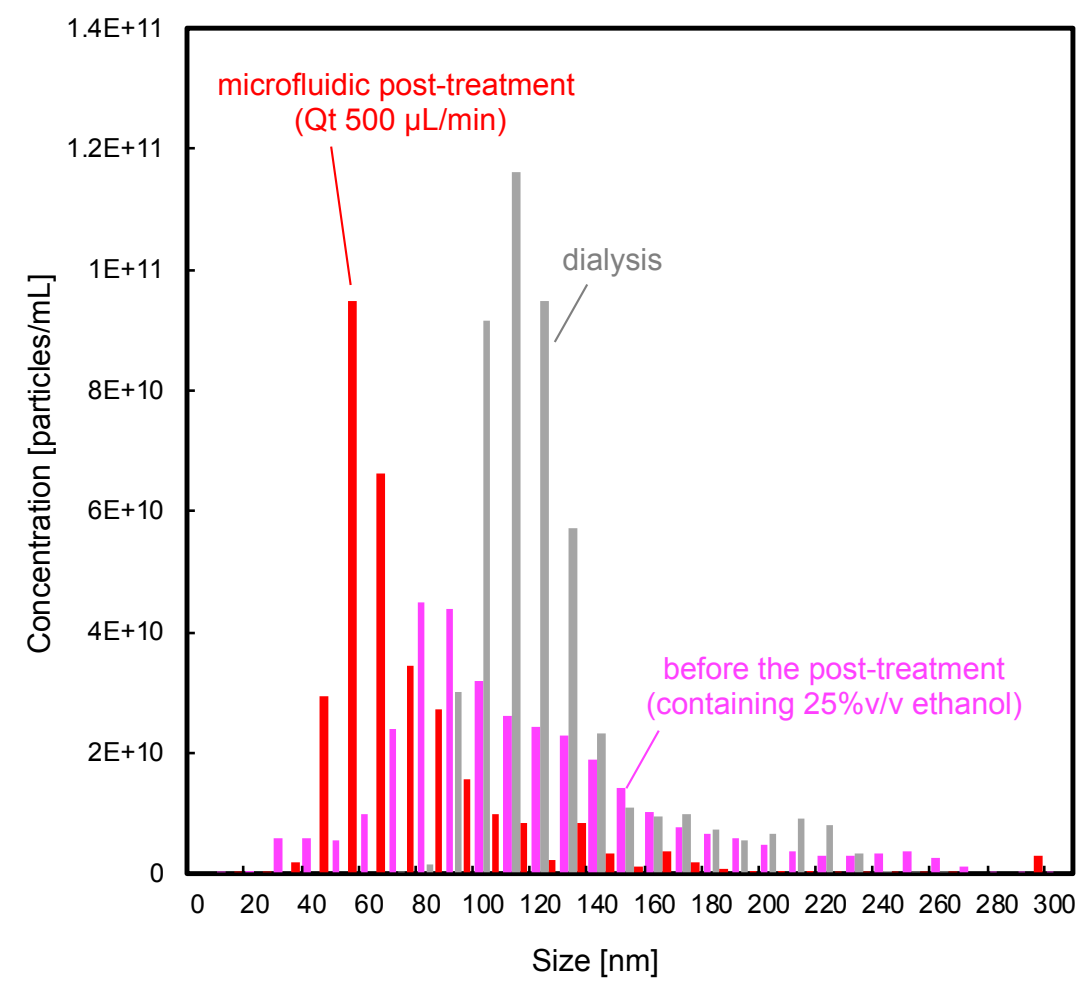

Figure S6. The size distributions of the POPC LNPs measured with the NTA (NanoSight NS300; Quantum Design, Tokyo, Japan). LNPs were prepared by introducing $13.4 \mathrm{mM}$ POPC/ethanol solution and saline into the simple baffle device at the total flow rate of 50 $\mu \mathrm{L} / \mathrm{min}$ and the FRR of 3 . The collected LNP suspension containing $25 \% \mathrm{v} / \mathrm{v}$ ethanol (magenta) was post-treated by the microfluidic method (red) or the dialysis method (grey). 


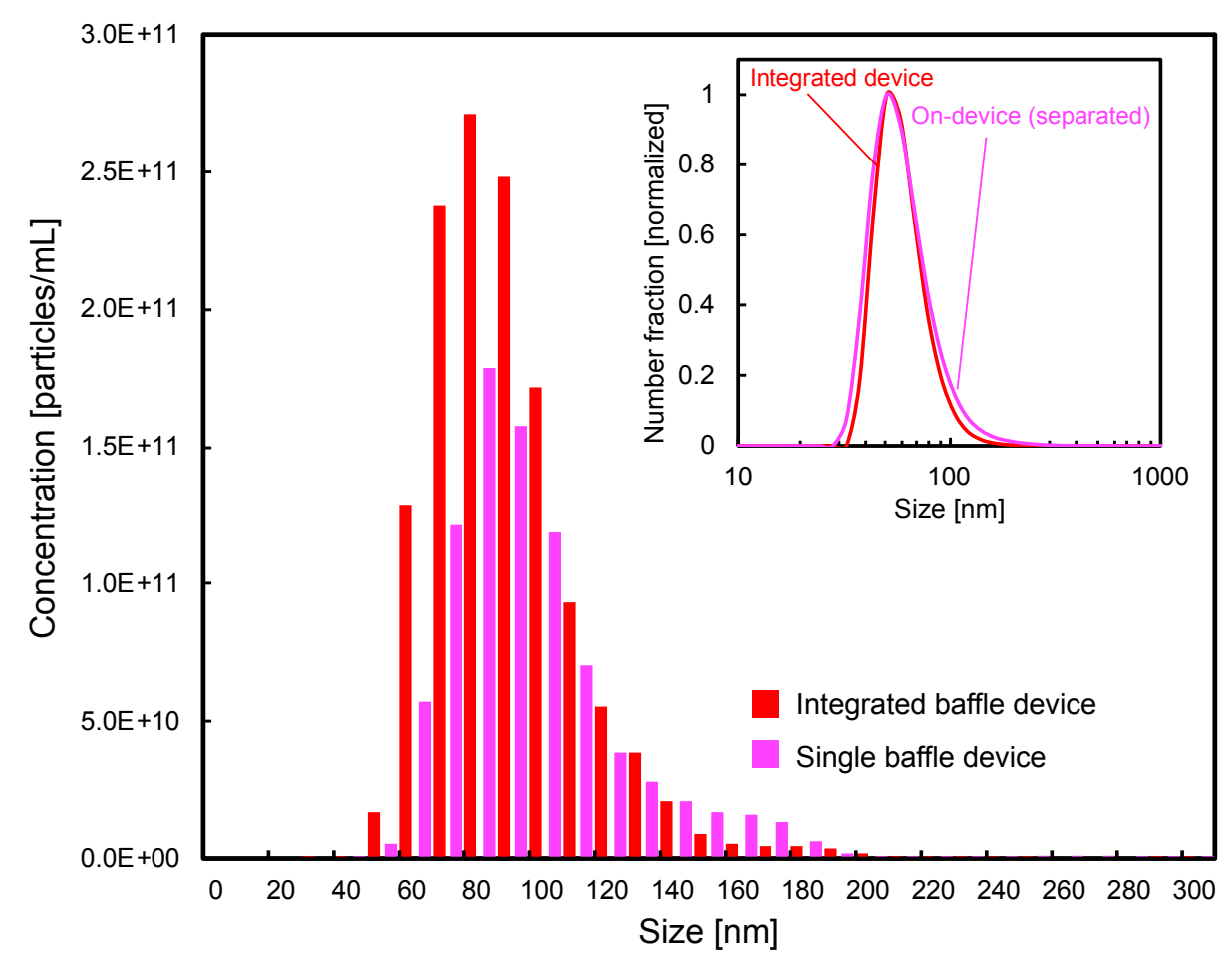

Figure S7. The size distributions of the POPC LNPs prepared under the high lipid concentration condition. The LNP sizes were measured with the NTA and using DLS (number-weighted, inset). We prepared $67 \mathrm{mM}$ POPC/ethanol solution and saline. These solutions were injected into the integrated baffle device for the mass production (red). To confirm the features of the integrated baffle device, the LNP suspension collected from the baffle device was re-introduced and post-treated using the same baffle device at the total flow rate of $500 \mu \mathrm{L} / \mathrm{min}$ (magenta). 


\section{Size and loaded siRNA stability of the post-treated YSK05-based LNPs}

Preparation process of siRNA-loaded YSK-05 LNPs was describes in the Experimental section in the main text (Preparation of siRNA encapsulated LNPs). The siRNA-loaded LNPs were treated by the dialysis, pipetting, and on-device post-treatment methods. For the on-device method, we prepared the siRNA-loaded YSK05-based LNP by using the simple baffle device at the total flow rate of $500 \mu \mathrm{L} / \mathrm{min}$ and FRR of 3. After the posttreatment, the LNP suspensions were dialyzed overnight against PBS followed by incubation at $4^{\circ} \mathrm{C}$ for a week.

Figure S8 (A) shows the number-weighted size distributions of the siRNA-loaded LNPs treated by the conventional and the on-device post-treatment methods. We did not observe any size-shift and leakage of siRNA after the incubation.
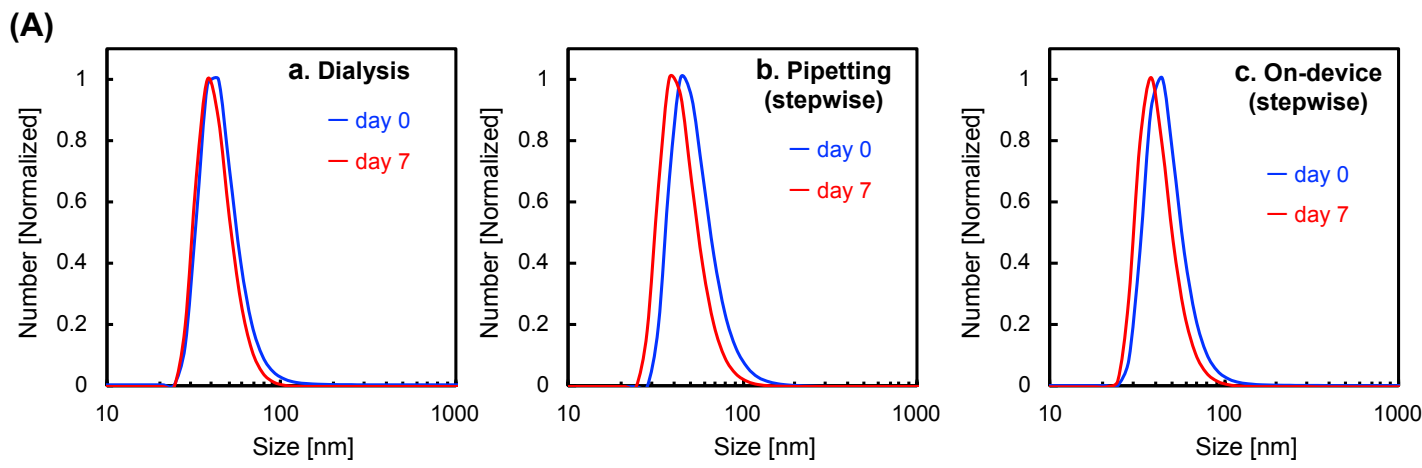

(B)

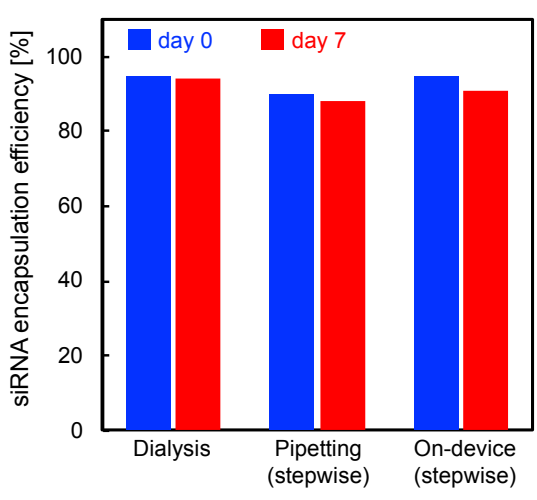


Figure S8. (A) Number-weighted size distributions of the siRNA-loaded LNPs treated by the conventional and the on-device post-treatment methods. For the pipetting and on-device post-treatment method, we employed the stepwise dilution. These distributions were normalized by the respective maximum intensity value. (B) siRNA encapsulation efficiency of the prepared LNPs by the different post-treatment methods. 


\section{Effect of the post-treatment methods on the siRNA release}

We examined the effect of the post-treatment methods on the siRNA release. siRNAloaded YSK-05 LNPs were prepared by the conventional dialysis and the stepwise ondevice post-treatment. The LNPs were prepared in the same manner as the main text (Preparation of siRNA encapsulated LNPs) except for the lipid composition (YSK05/ cholesterol/DMG-PEG2K: 50/50/1 mol\%).

Figure S9 shows number-weighted average sizes, siRNA-encapsulation efficiency and FVII activity of the siRNA-loaded LNPs treated by the conventional and the on-device post-treatment. The LNP size and siRNA encapsulation efficiency were almost the same values for the conventional dialysis method and the on-device post-treatment method. We confirmed higher gene silencing activity than $90 \%$ by both the conventional and the new post-treatment. This result indicates the post-treatment method does not affect the drug release.

(A)

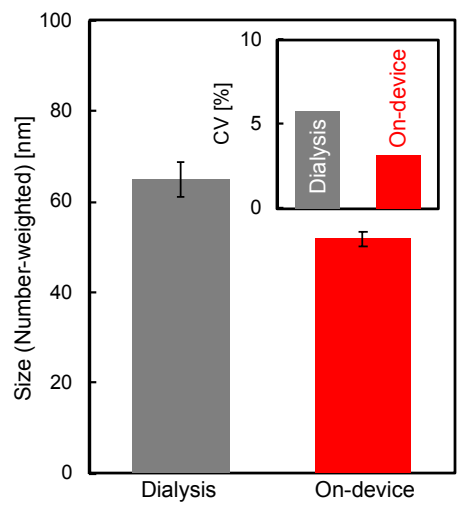

(B)

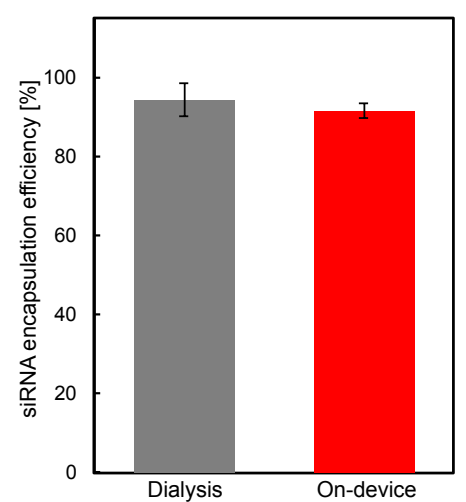

(C)

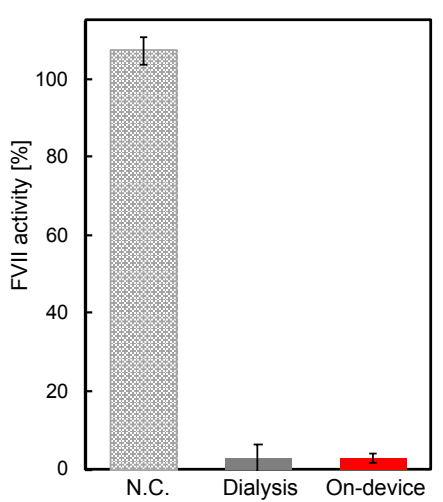

Figure S9. (A) average sizes, (B) the siRNA encapsulation efficiencies and (C) FVII activity of the siRNA-loaded YSK-05 LNPs post-treated by the conventional dialysis 
(gray) and the on-device stepwise dilution (red). The LNPs were intravenously administered to ICR mice at the dose of $0.5 \mathrm{mg}$ siFVII $/ \mathrm{kg}$. (C) Used with permission from the American Chemical Society. (Reference: Kimura, N.; Maeki, M.; Sato, Y.; Note, Y.; Ishida, A.; Tani, H.; Harashima, H.; Tokeshi, M. ACS Omega, 2018, 3, 5044-5051. 